\title{
Technical results, clinical efficacy and predictors of outcome of intercostal arteries embolization for hemothorax: a two-institutions' experience
}

\author{
Nicola Tamburini ${ }^{1}$, Nicole Carriel $^{2}$, Giorgio Cavallesco ${ }^{1}$, Laureano Molins ${ }^{2,3}$, Roberto Galeotti ${ }^{4}$, \\ Rudith Guzmán $^{2}$, Elisabetta Salviato ${ }^{4}$, David Sánchez-Lorente ${ }^{2}$, Elisa Maietti ${ }^{5,6}$, Pio Maniscalco ${ }^{1}$, \\ Marc Boada ${ }^{2}$
}

${ }^{1}$ Department of Morphology, Experimental Medicine and Surgery, Section of Chirurgia 1, Sant'Anna Hospital, University of Ferrara, Ferrara, Italy; ${ }^{2}$ General Thoracic Surgery Department, Respiratory Institute, Hospital Clínic de Barcelona, Universitat de Barcelona, Barcelona, Spain; ${ }^{3}$ Institut d'Investigacions Biomediques August Pi i Sunyer (IDIBAPS), Barcelona, Spain; ${ }^{4}$ Department of Radiology, Section of Interventional Radiology, Sant'Anna Hospital, University of Ferrara, Ferrara, Italy; ${ }^{5}$ Department of Biomedical and Neuromotor Sciences, University of Bologna, Bologna, Italy; ${ }^{6}$ Center for Clinical Epidemiology, Department of Medical Science, University of Ferrara, Ferrara, Italy

Contributions: (I) Conception and design: N Tamburini, M Boada; (II) Administrative support: R Galeotti, R Guzmán; (III) Provision of study materials or patients: E Salviato, P Maniscalco; (IV) Collection and assembly of data: N Carriel, G Cavallesco, L Molins; (V) Data analysis and interpretation: D Sánchez-Lorente, E Maietti; (VI) Manuscript writing: All authors; (VII) Final approval of manuscript: All authors.

Correspondence to: Nicola Tamburini. Department of Morphology, Experimental Medicine and Surgery, Section of Chirurgia 1, Sant' Anna Hospital, University of Ferrara, Via Aldo Moro 8, Ferrara 44124, Italy. Email: nicolatamburini@hotmail.it.

\begin{abstract}
Background: To evaluate the clinical efficacy and identify the predictors of outcome of intercostal arterial embolization for hemothorax caused by intercostal artery (ICA) injuries.

Methods: A retrospective multi-institutional study was conducted. Outcomes were analyzed in 30 consecutive patients presenting with hemothorax caused by active ICA hemorrhage undergoing transcatheter arterial embolization (TAE). Clinical and procedural parameters were compared between outcomes groups.

Results: Overall technical success rate was $87 \%(n=26)$. Among the 4 failed cases, 2 underwent repeated TAE and 2 underwent additional surgery. Overall 30-day mortality rate was 23\%. Low haemoglobin levels and haematocrit, hepatic comorbidities and more than one artery undergoing embolization increased technical failure rate significantly. Survival was poorer in patients with massive bleeding.

Conclusions: ICA embolization was found to be a safe and effective method in treating hemothorax caused by active ICA haemorrhage. Careful pre-embolization evaluation may be required for patient with low haemoglobin levels and haematocrit, hepatic comorbidities and active haemorrhage from more than one artery.
\end{abstract}

Keywords: Hemothorax; surgery; haemorrhage; embolization; thoracotomy; trauma

Submitted Feb 03, 2019. Accepted for publication Jul 08, 2019.

doi: $10.21037 /$ jtd.2019.10.27

View this article at: http://dx.doi.org/10.21037/jtd.2019.10.27

\section{Introduction}

Hemothorax caused by intercostal arteries (ICAs) bleeding is a life-threatening condition. For long time exploratory thoracotomy has been considered the therapeutic gold standard in patients with failed conservative treatment.
However, patients with severe associated injuries or serious medical comorbidities are poor candidates for surgical intervention. Furthermore, emergency surgery typically results in significant morbidity and low yield of finding the source of the haemorrhage (1). 


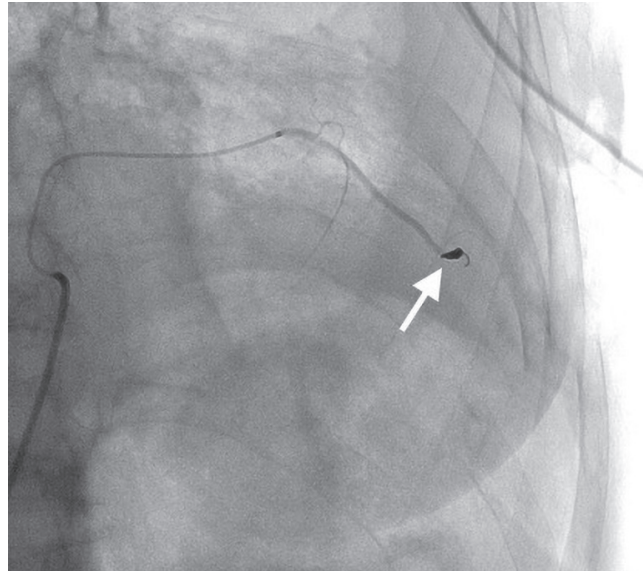

Figure 1 Selective angiogram using a microcatheter nearby bleeding site shows immediate post-intercostal artery embolization cessation of bleeding (white arrow).

For this reason, angiography and embolization of ICAs were introduced and approved gradually and have changed the treatment of ICAs haemorrhage as a safe procedure with few side effects. Transcatheter arterial embolization (TAE) provides a less invasive and safe therapeutic alternative for active bleeding from ICAs. In addition, it does not prohibit surgery if haemostasis is not carried out by embolization alone (2-8).

Knowledge of clinical and procedure-related features associated with embolization failure and the need for postTAE surgery would allow us to identify high-risk patients. Those could require closer clinical monitoring or prompt surgical intervention because of higher risk for technical failure. As far as we know, few reports have identified factors associated with technical failure and mortality in patients after selective embolization $(8,9)$.

The present multicenter study was conducted to identify factors that can predict outcomes and efficacy of ICA embolization as a primary therapeutic modality in the management of haemothorax.

\section{Methods}

A retrospective review of the medical records of all consecutive patients who underwent angiographic embolization because of hemothorax caused by ICA bleeding in two institutions from January 2009 to October 2017 was performed.

The study was approved by institutional ethics committee board of Hospital de Clinic of Barcelona (No.

\section{HCB/2019/0554).}

Patients demographics, past medical history, CT, angiography, pre- and post-embolization blood chemistries and clotting profiles, transfusion history, technical and clinical success rates, procedure-related complications and 30-day mortality rate were recorded. The number of RBC transfusions after TAE was calculated according to the number of transfusions after TAE.

Patients with ICA injuries who did not undergo embolization and/or had ICA injuries without hemothorax were excluded of the study.

Chest X-ray generally followed by CT scan of the chest were performed to detect the bleeding site, to rule out pseudo aneurysms and to identify other sources of haemorrhage than the ICA. Active haemorrhage from an ICA was defined as active extravasation of contrast agent in the arterial phase.

A multidisciplinary team including anesthesiologists, trauma surgeons, thoracic surgeons and interventional radiologists discussed each case. The treatment decision was made according to clinical, laboratory and CT findings.

Before the angiography, written informed consent was obtained and procedure-potential risks and benefits were disclosed with patients or relatives.

Interventions were performed either under general or local anesthesia, depending on the patients' status.

In all cases a right femoral approach was used. Each individual interventional radiologist made the choice of embolization method and material. Embolization agents used included gel foam pledgets, polyvinyl alcohol (PVA) particles, microcoils, or a combination of the above materials (Figure 1).

Total amount of red blood cells transfused (RBC), intensive care unit length of stay (ICU-LOS) and the total inpatient stay (LOS) for each patient were documented.

Technical success was defined as the immediate cessation of hemorrhage evaluated by completion angiography.

Patients underwent daily clinical and laboratory controls until discharge or transfer to another hospital or death. Furthermore, chest CT scan was obtained in the follow up to evaluate the effectiveness of embolization in short term.

\section{Statistical analysis}

In order to identify potential predictors of embolization outcome a comparison between success and no success groups was made. Furthermore, factors associated to 30-days mortality and risk for major surgical procedure 
Table 1 General characteristics of embolized patients

\begin{tabular}{|c|c|}
\hline Patients characteristics $(n=30)$ & $\mathrm{N}$ \\
\hline Age, years & $70[24-78]$ \\
\hline Gender, M:F (\%) & $22: 8$ [73.3:26.7] \\
\hline \multicolumn{2}{|l|}{ Comorbidities (\%) } \\
\hline Hypertension & $11[37]$ \\
\hline Ischemic heart disease & $12[40]$ \\
\hline Malignancy & $8[27]$ \\
\hline Liver impairment & $5[17]$ \\
\hline Diabetes mellitus & $3[10]$ \\
\hline COPD & $3[10]$ \\
\hline Renal impairment & $3[10]$ \\
\hline Antiplatelet therapy & $9[30]$ \\
\hline Anticoagulant therapy & $5[17]$ \\
\hline \multicolumn{2}{|l|}{ Causes of haemothorax (\%) } \\
\hline Traumatic & $18[60]$ \\
\hline latrogenic & $11[37]$ \\
\hline Thoracentesis & $4[36]$ \\
\hline Chest drain positioning & $2[18]$ \\
\hline Chest drain removal & $1[9]$ \\
\hline Cardiac surgery & $1[9]$ \\
\hline Hepatic radiofrequency & $2[18]$ \\
\hline Thoracotomy & $1[9]$ \\
\hline Spontaneous & $1[3]$ \\
\hline
\end{tabular}

COPD, chronic obstructive pulmonary diseases.

were analyzed. Continuous variables were described by calculating the median and inter-quartile range (IQR) and compared with the Wilcoxon-Mann-Whitney test, while for categorical factors counts and percentages were reported and the Fisher exact test was run. Analysis was done using Stata 13.0 for Windows (StataCorp, College Station, USA) and the level of significance was set to 0.05 .

\section{Results}

From 2009 through 2017,30 patients (22 men, 8 women) with a mean age of 70 years (range, $24-88$ years) presenting with hemothorax caused by an ICA as the site of haemorrhage underwent TAE of ICAs in the study centers. The most frequently comorbid condition was ischemic
Table 2 Treatment approach for hemothorax

\begin{tabular}{lc}
\hline Treatment approach & $\mathrm{N}$ \\
\hline TAE & $21[70]$ \\
Combined & $3[10]$ \\
Coils only & $3[10]$ \\
Gelatin sponge only & $3[10]$ \\
PVA particles only & \\
Number of arteries embolised & $16[53]$ \\
1 artery & $5[17]$ \\
2 arteries & $7[23]$ \\
3 arteries & $2[7]$ \\
4 arteries & \\
After-TAE procedures & $12[40]$ \\
Chest drain & $7[23]$ \\
Chest drain + thoracotomy & $6[20]$ \\
Chest drain + VATS & $2[7]$ \\
Exploratory thoracotomy & $7[23]$ \\
Outcome & \\
Mortality & \\
\hline TAE, transcather ateriat & \\
\hline
\end{tabular}

TAE, transcatheter arterial embolization; PVA, polyvinyl alcohol; VATS, video-assisted thoracoscopic surgery.

heart disease $(\mathrm{n}=12 ; 40 \%)$ followed by hypertension $(\mathrm{n}=11$; $37 \%)$ and malignancy $(\mathrm{n}=8 ; 27 \%)$.

The causes of bleeding included trauma in 18 patients $(60 \%)$, iatrogenic in 11 patients $(37 \%)$ and haemorrhage occurred spontaneously in 1 patient $(3 \%)$. Iatrogenic ICA lesions were caused by thoracentesis in 4 cases $(36 \%)$, liver radiofrequency in 2 patients (18\%), chest tube positioning in 2 cases (18\%), thoracotomy, chest tube removal and cardiac surgery in one case (9\%) as reported in Table 1.

Twenty-six patients (87\%) underwent chest CT scan showing arterial blush.

In half of the cases registered only one artery was needed to be embolized (16 cases, 53\%). Embolization was mainly done using a combination of embolic agents-coils, spongostan and/or PVA (21 cases, 70\%). No procedurerelated major complications occurred. Diagnostic and treatment details are summarized in Table 2.

Primary technical success was achieved in 26 patients (87\%). Two patients required repeated TAE to achieve bleeding control: one case had bleeding from a mammary 
Table 3 Predictors of technical failure after TAE

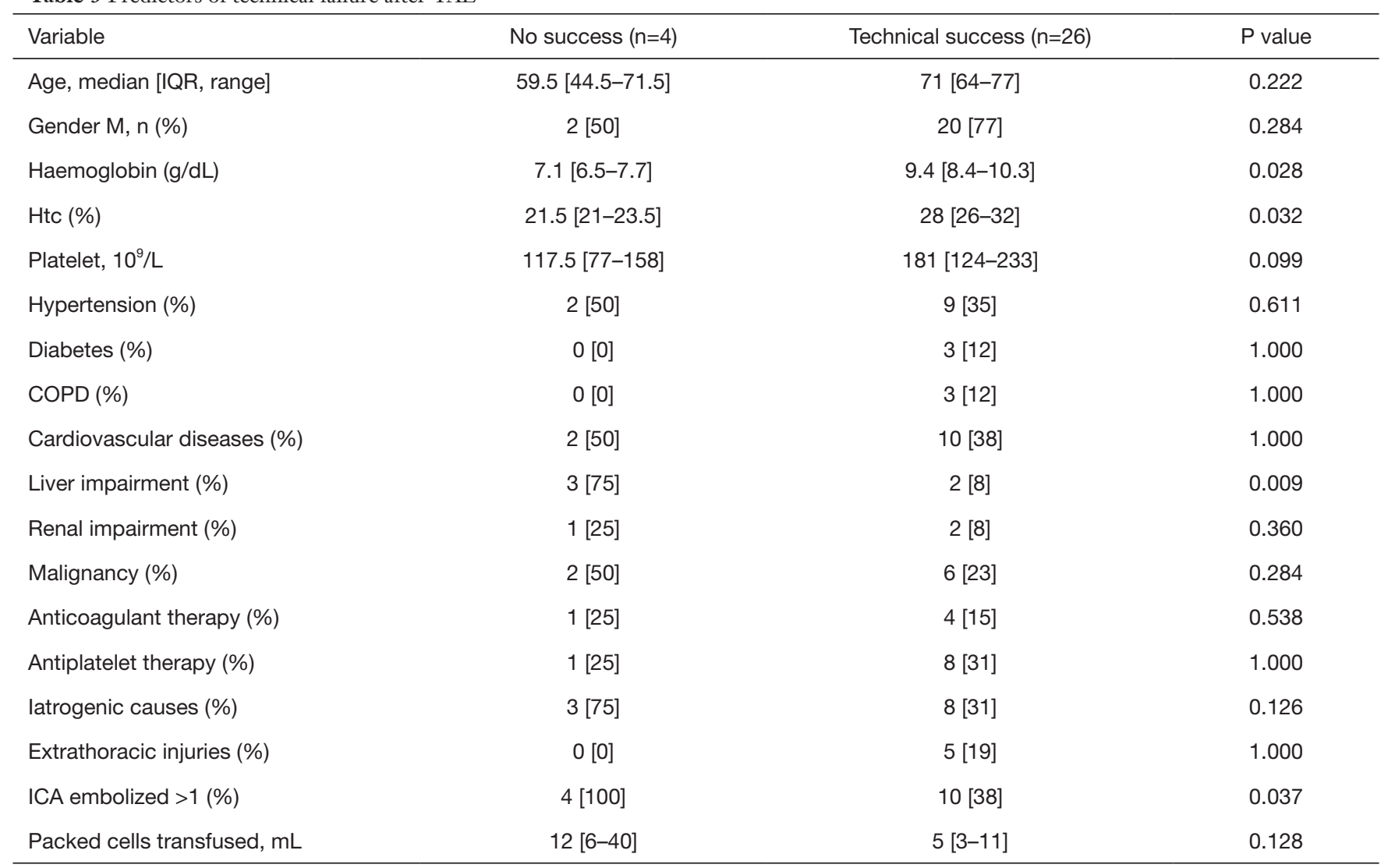

TAE, transcatheter arterial embolization; IQR, inter-quartile range; COPD, chronic obstructive pulmonary diseases; ICA, intercostal artery.

artery dependent intercostal branch not previously detected. The other one showed bleeding from a different ICA. The remaining two patients underwent exploratory thoracotomy. The analysis of parameters associated with technical efficacy is summarized in Table 3. Using univariate and multivariable logistic regression models, low haemoglobin levels, low haematocrit, hepatic comorbidities and more than one artery undergoing embolization were the factors associated to technical failure.

Overall, 27 (90\%) patients required surgical treatment after the interventional procedure. Twelve patients (40\%) needed chest tube only, in 13 patients (43\%) surgery was carried out for retained hemothorax either by video-assisted thoracoscopic surgery (VATS) (7 cases, $23 \%$ ) or thoracotomy (6 cases, $20 \%$ ) and in 2 patients (7\%) exploratory thoracotomy was necessary to manage incontrollable bleeding.

The overall 30 -days mortality rate was $23 \%(n=7)$ : the causes of death were massive bleeding and associated severe comorbidities in 3 patients (43\%), pneumonia and pulmonary thromboembolism in 1 patient (14\%), 1 patient died for pneumonia and haemoptysis (14\%), 1 patient for massive bleeding (14\%) and 1 patient for myocardial ischemia (14\%). The analysis of factors associated to 30-days mortality is shown in Table 4. The analysis revealed that the number of RBC transfusions was the only factor related to death, liver impairment showed a marginal but non-statistically significant relationship $(\mathrm{P}=0.068)$.

The analysis of factors related to the risk of undergoing major surgical procedure (both VATS or thoracotomy) didn't find any significant association.

\section{Discussion}

TAE constitutes an established endovascular management for a broad range of indications and is also widely performed for the emergency management of acute hemorrhage. Several studies reported the efficacy of embolization for acute postoperative bleeding, spontaneous hemorrhage and post-traumatic hemorrhage (10-12). 
Table 4 Predictors of 30-day mortality after TAE

\begin{tabular}{|c|c|c|c|}
\hline Variable & 30-day mortality, NO ( $n=23)$ & 30-day mortality, YES $(n=7)$ & $P$ value \\
\hline Gender M, n (\%) & 16 [70] & $6[86]$ & 0.638 \\
\hline $\mathrm{Hb}(\mathrm{g} / \mathrm{L})$ & $8.8[8.1-10.1]$ & $8.5[6.2-11.6]$ & 0.731 \\
\hline $\mathrm{Htc}(\%)$ & 27 [25-31] & 27 [20-35] & 0.805 \\
\hline Hypertension (\%) & 9 [39] & $2[29]$ & 1.000 \\
\hline Diabetes (\%) & $3[13]$ & $0[0]$ & 1.000 \\
\hline COPD (\%) & $2[9]$ & $1[14]$ & 1.000 \\
\hline Cardiovascular diseases (\%) & $10[43]$ & 2 [29] & 0.669 \\
\hline Malignancy (\%) & $6[26]$ & $2[29]$ & 1.000 \\
\hline Anticoagulant therapy (\%) & $5[22]$ & $0[0]$ & 0.304 \\
\hline Antiplatelet therapy (\%) & $8[35]$ & $1[14]$ & 0.393 \\
\hline latrogenic causes (\%) & 15 [65] & $4[57.1]$ & 1.000 \\
\hline Extrathoracic injuries (\%) & $4[17]$ & $1[14.3]$ & 1.000 \\
\hline ICA embolized >1 (\%) & 10 [43] & $4[57]$ & 0.675 \\
\hline Packed cells transfused $\mathrm{mL}$ & $4[3-8]$ & $12[10-30]$ & 0.0013 \\
\hline Technical failure (\%) & $3[13]$ & $1[14]$ & 1.000 \\
\hline
\end{tabular}

TAE, transcatheter arterial embolization; IQR, inter-quartile range; COPD, chronic obstructive pulmonary diseases; ICA, intercostal artery.

In our institutions, traumatic ICA laceration was most often the cause of the bleeding in $60 \%$ of cases. Selective catheterization was started on the target artery defined by CT. Other arteries were not catheterized unless there was suspicion of additional sites of bleeding. In our opinion CT scan should be obtained whenever possible to identify hemothorax origin and to plan endovascular treatment.

The type of embolic material did not seem to affect the clinical outcomes or recurrence rates. Therefore, various combinations of embolic materials are commonly used (70\% of cases) depending on interventional radiologist's choice based on clinical and technical aspects.

According to previous studies $(8,9)$, our study showed no major complications such as spinal cord ischemia: these results show good safety profile of the procedure with low incidence of clinically significant complications.

Only a few studies reported embolotherapy in patients with acute hemorrhage from ICAs (1-9). However, there is widespread agreement that TAE is a reliable and feasible therapeutic alternative to thoracotomy to control intrathoracic arterial bleeding. In our study, emergency intercostal TAE was an effective approach in patients with acute hemorrhage. Our study, which included a larger study population, had a success rate of $87 \%$, which is comparable to those previously reported. Chemelli et al. in a series of 24 patients described an immediate success of $87 \%$ (8). As described by Stampfl et al. in their series of 19 (85\%) (9), and Hagiwara et al. $(100 \%)$ in 5 patients who underwent TAE (7).

Since none of the studies mentioned above analyzed potential predictors of TAE failure, there are few known clinical risk factors for failure in ICAs TAE patients. Our series highlighted that some factors could predict the efficacy of the endovascular procedure. Low hemoglobin levels and low hematocrit were confirmed as significant risk factors for technical failure. We suggest that the 
results may simply indicate that a more severe initial bleed associated with poorer patient's general condition and less effective coagulation. In consequence, increased risk of failure. Likewise, patients with chronic liver disease, which is known to cause coagulation abnormalities, were also shown to have a higher incidence of failure. Other authors identified underlying chronic liver disease as a risk factor for rebleeding after bronchial arteries embolization due to pulmonary tuberculosis (13). Although we analysed anticoagulant and antiplatelet agents, we did not identify a trend toward increasing failure risk for any individual agent. Analysing the angiographic features, the number of arteries undergoing embolization was found associated with a higher failure rate. Patients with one artery embolized had a better outcome in term of bleeding control compared to patients with two arteries or more undergoing TAE.

Despite the high success rate described before, $90 \%$ of patients required some kind of surgery. However, 12 of the cases needed a minor procedure for chest tube insertion and 13 patients needed a non-emergent exploration of the chest cavity to remove clotted haemothorax. Consequently, only in two patients there was a need for an emergent surgical exploration for bleeding control. This means that patient optimization could be done before surgery and an even less invasive approach (VATS) can be carried out in some cases. This all reducing surgical associated morbidity and mortality.

In our study the 30 -day mortality rate was $23 \%$. Similar to previously published data $(1,8,9)$. Stampfl et al. (9) described a 30 -day mortality rate of $21 \%$. In another study with a higher incidence of cases with iatrogenic ICA injuries the total cumulative mortality rate was $37.5 \%$ (8).

In nearly half of the cases ( $43 \%)$, death was related to multiple organ failure, most likely as a result of haemodynamic shock in association with the patient's comorbid conditions, despite technically successful management of the bleeding. The number of RBC transfusions was the only factor related to death. In our opinion, this may be mainly attributed to a more severe bleeding.

\section{Limitations}

This study was unavoidably limited by its retrospective analysis and by the small sample size. Furthermore, the study population was heterogeneous, including patients with traumatic as well as iatrogenic injuries. Likewise, the angiographic embolization procedure was performed using different embolic materials based on the radiologist's preference.

Although these limitations are significant, this study remains important in looking at the effectiveness and safety of selective embolization in the definitive management of hemothorax by ICAs bleeding. It has also identified various factors that could help predict patients who have a higher risk for rebleeding that requires surgical intervention, and maybe even the type of surgery to be performed.

\section{Conclusions}

Embolization is a safe and effective measure to arrest bleeding in patients with haemothorax caused by ICAs injuries.

Patients with severe bleeding, more than one artery injured and/or liver impairment might be cautiously considered because of higher risk of technical failure. On those patients' straightforward surgery could be indicated in order to reduce risk of death.

\section{Acknowledgments}

None.

\section{Footnote}

Conflicts of Interest: The authors have no conflicts of interest to declare.

Ethical Statement: The authors are accountable for all aspects of the work in ensuring that questions related to the accuracy or integrity of any part of the work are appropriately investigated and resolved. The study was approved by institutional ethics committee board of Hospital de Clinic of Barcelona (No. HCB/2019/0554). Written informed consent was obtained from the patient for publication of this manuscript and any accompanying images.

\section{References}

1. Carrillo EH, Heniford BT, Senler SO, et al. Embolization therapy as an alternative to thoracotomy in vascular injuries of the chest wall. Am Surg 1998;64:1142-8.

2. Kessel B, Alfici R, Ashkenazi I, et al. Massive hemothorax caused by intercostal artery bleeding: selective embolization may be an alternative to thoracotomy in selected patients. 
Thorac Cardiovasc Surg 2004;52:234-6.

3. Aoki T, Okada A, Tsuchida M, et al. Ruptured intercostal artery pseudoaneurysm after blunt thoracic trauma. Thorac Cardiovasc Surg 2003;51:346-7.

4. Barbaric ZL, Luka NL. Angiographic demonstration and transcatheter embolic control of post-traumatic intercostal arterial hemorrhage. Surgery 1977;81:409-2.

5. Bluebond-Langner R, Pinto PA, Kim FJ, et al. Recurrent bleeding from intercostal arterial pseudoaneurysm after retroperitoneal laparoscopic radical nephrectomy. Urology 2002;60:1111.

6. Casas JD, Perendreu J, Gallart A, et al. Intercostal artery pseudo- aneurysm after a percutaneous biliary procedure: diagnosis with CT and treatment with transarterial embolization. J Comput Assist Tomogr 1997;21:729-30.

7. Hagiwara A, Yanagawa Y, Kaneko N, et al. Indications for transcatheter arterial embolization in persistent hemothorax caused by blunt trauma. J Trauma 2008;65:589-94.

8. Chemelli AP, Thauerer M, Wiedermann F, et al.

Transcatheter arterial embolization for the management of iatrogenic and blunt traumatic intercostal artery injuries. J Vasc Surg 2009;49:1505-13.

9. Stampfl U, Sommer CM, Bellemann N, et al. Emergency embolization for the treatment of acute hemorrhage from intercostal arteries. Emerg Radiol 2014;21:565-70.

10. Sommer CM, Stampfl U, Bellemann N, et al. Patients with life-threatening arterial renal hemorrhage: CT angiography and catheter angiography with subsequent superselective embolization. Cardiovasc Intervent Radiol 2010;33:498-508.

11. Sunga KL, Bellolio MF, Gilmore RM, et al. Spontaneous retroperitoneal hematoma: etiology, characteristics, management, and outcome. J Emerg Med 2012;43:e157-61.

12. Grossi W, Maniscalco P, Quarantotto F, et al. Endovascular and thoracoscopic treatment for post-lobectomy hemothorax. Eur J Cardiothorac Surg 2012;41:456.

13. Kim WS, Lee SJ, Ryu YJ, et al. Prognosis and Predictors of Rebleeding After Bronchial Artery Embolization in Patients with Active or Inactive Pulmonary Tuberculosis. Lung 2015;193:575-81.
Cite this article as: Tamburini N, Carriel N, Cavallesco G, Molins L, Galeotti R, Guzmán R, Salviato E, Sánchez-Lorente D, Maietti E, Maniscalco P, Boada M. Technical results, clinical efficacy and predictors of outcome of intercostal arteries embolization for hemothorax: a two-institutions' experience. J Thorac Dis 2019;11(11):4693-4699. doi: 10.21037/jtd.2019.10.27 